\title{
Flash glucose monitoring: objective, not self-referential, outcomes are needed. Reply to Warren RE [letter]
}

\author{
Jan Bolinder ${ }^{1}$ - Per Oskarsson ${ }^{1} \cdot$ Ramiro Antuna ${ }^{2} \cdot$ Petronella Geelhoed-Duijvestijn $^{3}$ • Jens Kröger ${ }^{4}$. \\ Raimund Weitgasser ${ }^{5,6}$
}

Received: 16 April 2018 / Accepted: 19 April 2018 / Published online: 2 June 2018

(C) The Author(s) 2018

Keywords Clinical diabetes $\cdot$ Devices $\cdot$ Hypoglycaemia $\cdot$ Insulin therapy

\author{
Abbreviation \\ IMPACT Novel Glucose-Sensing Technology \\ and Hypoglycaemia in Type 1 Diabetes: \\ a Multicentre Non-masked \\ Randomised Controlled Trial
}

To the Editor: We thank Dr Roderick Warren for his letter [1] regarding our subgroup analysis of the Novel GlucoseSensing Technology and Hypoglycaemia in Type 1 Diabetes: a Multicentre, Non-masked, Randomised Controlled Trial (IMPACT; NCT02232698) [2]. In line with the accepted practice in continuous glucose monitoring studies, the trial was designed to compare individuals using the FreeStyle Libre system (Abbott Diabetes Care, Witney, UK) for glucose monitoring with those using the conventional technique, self-monitoring blood glucose

Jan Bolinder

jan.bolinder@ki.se

1 Department of Medicine, Karolinska University Hospital Huddinge, Karolinska Institute, 14186 Stockholm, Sweden

2 Department of Medicine, Clinica Diabetologica, Gijon, Spain

3 Department of Internal Medicine, Haaglanden Medisch Centrum, Den Haag, the Netherlands

4 Department of Diabetes, Zentrum für Diabetologie Hamburg Bergedorf, Hamburg, Germany

5 Department of Medicine, Wehrle-Diakonissen Hospital, Salzburg, Austria

6 1st Department of Medicine, University Hospital of Paracelsus, Medical Private University, Salzburg, Austria
(SMBG). The main glycaemic outcome measures with this type of study design require sensor glucose results to be visible to the intervention group but blinded in the control group. Warren reiterates a valid point regarding using the same device for the treatment and assessment of an outcome. This was indeed noted in our original study article [3]; however the general consensus is that there is no practical alternative to this approach [4].

With regard to the reliability of the sensor recordings, the accuracy of FreeStyle Libre in the low glucose range (capillary reference $<4.4 \mathrm{mmol} / 1$ [ $80 \mathrm{mg} / \mathrm{dl}]$ ), calculated from raw data collected by Bailey et al [5] had a mean bias of $0.2 \mathrm{mmol} / \mathrm{l}$ $(2.9 \mathrm{mg} / \mathrm{dl})$ and a mean absolute difference (MAD) of $0.6 \mathrm{mmol} / \mathrm{l}(10.4 \mathrm{mg} / \mathrm{dl})$. In the IMPACT study baseline phase, mean bias was $0.0 \mathrm{mmol} / \mathrm{l}(0.3 \mathrm{mg} / \mathrm{dl})$ and MAD $0.6 \mathrm{mmol} / \mathrm{l}$ (10.6 mg/dl) for capillary glucose $<4.4 \mathrm{mmol} / \mathrm{l}$. A recent head to head study supports the accuracy of FreeStyle Libre vs two continuous glucose monitoring (CGM) devices in the hypoglycaemic range [6].

Warren states that studies like this require externally valid outcomes such as $\mathrm{HbA}_{1 \mathrm{c}}$ or severe hypoglycaemia but does not suggest a viable alternative outcome that could have been utilised for this study. $\mathrm{HbA}_{1 \mathrm{c}}$ did not increase in the intervention group, which should be viewed positively in the context of a study population with extremely well-controlled blood glucose, reduced hypoglycaemia and a final mean $\mathrm{HbA}_{1 \mathrm{c}}$ of $53.0 \mathrm{mmol} / \mathrm{mol}$. Severe hypoglycaemia is indeed very rare, with six instances reported in the treatment phase of this study (two in the intervention group and four in the control group), and therefore much larger numbers of patients would need to be studied over long periods to detect a clinically relevant improvement. Notably, there was a $30 \%$ reduction in the frequency of participant-reported symptomatic hypoglycaemia in the final 2 weeks $(p=0.0063)$. Hence, in our view, this similar reduction in symptomatic 
hypoglycaemia is corroborating evidence for the validity of the primary outcome result.

Acknowledgements The authors thank Z. Welsh (Statistics, Abbott Diabetes Care, Witney, UK).

Funding This study was funded by Abbott Diabetes Care and by grants from the Swedish Diabetes Association and the Diabetes Research Program at Karolinska Institutet.

Duality of interest JB has received honoraria for consulting or lecture fees from Abbott Diabetes Care, AstraZeneca, Insulet Corporation, Integrity Applications, Novo Nordisk, and Sanofi-Aventis. RA has received honoraria for lecture fees for Abbott Diabetes Care. PG-D has received lecture honoraria from Abbott Diabetes Care and Medtronic and served on advisory boards for Medtronic. JK has received personal fees from Abbot Diabetes Care, personal fees from Abbott Diabetes Care during the conduct of this study; personal fees from Lilly, Novo Nordisk, Berlin Chemie, Medtronic, Sanofi, MSD and AstraZeneca. RW received lecture honoraria and serves on advisory boards for Abbott Diabetes Care, BoehringerIngelheim, Eli Lilly, Merck Sharp \& Dohme, Novo Nordisk, Roche Diabetes Care, Sanofi and Servier; and has received unrestricted study grants from Eli Lilly, Novo Nordisk, and Sanofi. PO declares that there is no duality of interest associated with his contribution to this manuscript.

Contribution statement JB wrote the first draft of the response letter and worked collaboratively with the co-authors to discuss and review this response. All authors reviewed and approved the final draft and approved the submission.

Open Access This article is distributed under the terms of the Creative Commons Attribution 4.0 International License (http:// creativecommons.org/licenses/by/4.0/), which permits unrestricted use, distribution, and reproduction in any medium, provided you give appropriate credit to the original author(s) and the source, provide a link to the Creative Commons license, and indicate if changes were made.

\section{References}

1. Warren RE (2018) Flash glucose monitoring: objective, not self-referential, outcomes are needed. Diabetologia. https://doi.org/10.1007/ s00125-018-4608-0

2. Oskarsson P, Antuna R, Geelhoed-Duijvestijn P, Kroger J, Weitgasser R, Bolinder J (2018) Impact of flash glucose monitoring on hypoglycaemia in adults with type 1 diabetes managed with multiple daily injection therapy: a pre-specified subgroup analysis of the IMPACT randomised controlled trial. Diabetologia 61:539-550

3. Bolinder J, Antuna R, Geelhoed-Duijvestijn P, Kröger J, Weitgasser R (2016) Novel glucose-sensing technology and hypoglycemia in type 1 diabetes: a multicentre, non-masked, randomised controlled trial. Lancet 388:2254-2263

4. Maahs DM, Buckingham B, Castle J et al (2016) Outcome measures for artificial pancreas clinical trials: a consensus report. Diabetes Care 39:1175-1179

5. Bailey T, Bode BW, Christiansen MP et al (2015) The performance and usability of a factory-calibrated flash glucose monitoring system. Diabetes Technol Ther 17:787-794

6. Aberer F, Hajnsek M, Rumpler M et al (2017) Evaluation of subcutaneous glucose monitoring systems under routine environmental conditions in patients with type 1 diabetes. Diabetes Obes Metab 19:1051-1055 